\title{
$\mathrm{R} C \& \mathrm{C}$
}

REVISTA DE CONTABILIDADE E CONTROLADORIA

\section{A INFLUÊNCIA DA POLÍTICA DE DIVIDENDOS SOBRE A VOLATILIDADE DAS AÇÕES}

\section{THE INFLUENCE OF DIVIDEND POLICY ON THE VOLATILITY OF SHARES}

Recebido em 01.06.2014 | Aceite final em 04.11.2015 |

Nota: este artigo foi aceito pelo Editor Jorge Eduardo Scarpin e passou por uma avaliação double

\section{CLÁUDIO PILAR DA SILVA JÚNIOR} Doutorando em Administração | Universidade Federal da Paraíba | Professor da Universidade Federal de Sergipe | Av. Marechal Rondon, s/n | Jardim Rosa Elze | São Cristóvão/SE | CEP 49100-000 | Telefone: (83) 3233-5155 | Email: claudiopilar.adm@gmail.com|

\section{MÁRCIO ANDRÉ VERAS MACHADO}

Doutor em Administração | Professor do Programa de Pós-Graduação em Administração (PPGA) da Universidade Federal da Paraíba e do Programa Multiinstitucional e Inter-regional de Pós-Graduação em Ciências Contábeis UnB/UFPB/UFRN | Cidade Universitária - Campus I. Castelo Branco| CEP: 58059900 | João Pessoa/PB | Tel.: (83) 3216-7492 | E-mail: mavmachado@hotmail.com

\section{RESUMO}

De modo a analisar a relação entre a política de dividendos e o risco das ações, mensurada pela variação nos preços, este artigo teve por objetivo verificar como a volatilidade do preço das ações negociadas na BM\&FBOVESPA é influenciada pela política de dividendos das corporações. Para o desenvolvimento do estudo, fez-se uso da análise de regressão em corte transversal, onde a amostra final é composta por 914 empresas não financeiras, pagadoras de dividendos e listadas na BM\&FBOVESPA, no período de 2000 a 2012. Para examinar a relação entre a política de dividendos e a volatilidade das ações, foram utilizadas duas variáveis explicativas: dividend yield e o índice payout. Adicionalmente, utilizou-se como variáveis de controle a variável tamanho, a volatilidade dos lucros, a dívida de longo prazo e o crescimento dos ativos. O modelo econométrico utilizou a abordagem proposta por Baskin (1989). Como resultados principais, verificou-se que a política de dividendos no Brasil demonstrou-se significante na determinação da volatilidade, evidenciando que quanto mais elevado o dividend yield, menor o risco a ser enfrentado pelo acionista. Adicionalmente, como esperado, observou-se que o tamanho influenciou negativamente as oscilações do preço das ações, enquanto que a dívida de 
longo prazo e o crescimento dos ativos demonstraram um efeito direto sobre a volatilidade do preço das ações. Já as variáveis índice payout e volatilidade dos lucros não apresentaram significância estatística. Por fim, esta pesquisa contribui com novas evidências empíricas, pois fornece informações relevantes sobre o risco para os investidores que utilizam a estratégia de dividendos para investir no mercado acionário risco para os investidores que utilizam a estratégia de dividendos para investir no mercado acionário.

Palavras-chave: Política de dividendos. Investimento. Volatilidade.

\begin{abstract}
In order to analyze the relationship between the dividend policy and the risk of stocks, measured by the change in prices, this article aims at verifying how the volatility of the price of the shares traded on the BM \& FBOVESPA is influenced by corporate dividend policy. To develop the study, made use of regression analysis in cross-section, where the final sample consists of 914 non-financial companies, dividend payers and listed on the BM\&FBOVESPA, in the period 2000-2012. To examine the relationship between dividend yield and payout ratio: the dividend policy and the volatility of the stock, two explanatory variables were used. Additionally, it was used as control variables the variable size, earnings volatility, the longterm debt and asset growth. The econometric model uses proposed by Baskin (1989) approach. As main results, it was found that the dividend policy in Brazil proved to be significant in determining the volatility, showing that the higher the dividend yield, the lower the risk to be faced by the shareholder. Additionally, as expected, it was observed that the size negatively influenced the fluctuations of the stock price, while the longterm debt and asset growth demonstrated a direct effect on the volatility of the stock price. The variables payout ratio and earnings volatility are not statistically significant. Finally, this research contributes new empirical evidence, it provides relevant information about the risk for investors who use the dividend strategy to invest in the stock market.
\end{abstract}

Keywords: Dividend Policy. Investment. Volatility.

\title{
1 INTRODUÇÃO
}

Cinquenta anos após o artigo desenvolvido por Miller e Modiglianni (1961), ainda não se tem uma decisão concreta a respeito da influência da política de dividendos das organizações. Diversos pesquisadores procuraram analisar, nesse período, a principal questão relacionada à política de dividendos: saber discernir sobre o grau de relevância ou não para as organizações.

A política de dividendos refere-se às decisões relacionadas ao que fazer com o lucro líquido da empresa, ou seja, distribuí-los aos acionistas, na forma de dividendos, ou reinvesti-los, como fonte de financiamentos de projetos geradores de valor. Adicionalmente, além da distribuição de proventos, a política de dividendos poderá ocorrer por meio de processos relacionados às ações, quais sejam:

- Bonificação - são emissões adicionais, sem custos para os acionistas, na proporção de seus investimentos atuais; 
- Split ou desdobramento - estratégia utilizada pelas empresas com o objetivo principal de melhorar a liquidez de suas ações, com o aumento no número das mesmas e uma redução proporcional do valor nominal;

- Recompra de ações - processo que permitem as empresas devolverem recursos aos acionistas e ainda permanecer flexível em períodos futuros.

No Brasil, a forma de distribuição do lucro líquido poderá ocorrer de duas maneiras: dividendos e/ou juros sobre capital próprio. A base legal sobre a distribuição de dividendos está contida na Lei no 6.404/76, atualizada pela Lei 10.303/01, onde se estabelece que o estatuto da própria sociedade por ações é quem determina qual a parcela do lucro a ser distribuída como dividendo obrigatório, não sendo permitido ser inferior a $25 \%$ do lucro líquido ajustado.

Além disso, nos casos em que o assunto for omisso e couber à assembleia geral decidir sobre a matéria, os acionistas têm direito ao recebimento de metade do lucro líquido ajustado acrescido da reversão da parcela realizada dos lucros anteriormente registrados na reserva de lucros a realizar, se não absorvidos por prejuízos. Por outro lado, apesar de toda regulamentação quanto à distribuição de dividendos, verifica-se que algumas empresas são relutantes à sua distribuição e utilizam o lucro líquido como fonte de financiamento de projetos, restando aos acionistas apenas o ganho de capital (LEAL; SAITO, 2003).

Com relação aos Juros Sobre Capital Próprio (JSCP), o mesmo foi criado e regulado pela Lei no 9.249/95 em substituição à correção monetária dos balanços no Brasil. O maior benefício para as organizações que remuneram seus acionistas por esse método é a economia do imposto de renda. Entretanto, embora a distribuição por JSCP seja mais benéfica para as empresas, há muitas organizações que possuem grande disponibilidade de JSCP e não utilizam em suas políticas de distribuição, ocasionando a destruição de valor (MOTA; EID JUNIOR, 2007)

Em geral, os estudos relacionados à política de dividendos estão mais direcionados à influência destes sobre o retorno das ações. De modo contrário, este estudo volta-se para a relação entre a política de dividendos e o risco das ações, mensurada pela variação nos preços.

As principais pesquisas que relacionam a influência do dividend yield sobre a variabilidade dos preços das ações foram desenvolvidas por Harkavy (1953), Friend e Puckett (1964), Litzenberger e Ramaswamy (1982), Baskin (1989), Allen e Rachim (1996), Nishat e Irfan (2001), Rashid e Rahman (2008), Nazir et al. (2010), Hussainey, Mgbame e Chijoke-Mgbame (2011) e Hashemijoo, Ardekani e Younesi (2012). Embora esses estudos não apresentem uma conclusão definitiva sobre o assunto em questão, eles demonstraram que há uma relação linear entre as duas variáveis, onde, em alguns casos, a variável utilizada para explicar a relação com o preço, o dividend yield e/ou índice payout, não apresentam significância estatística.

Apesar de inúmeras pesquisas relacionarem a política de dividendos como forma de estratégia de investimento, obtenção de retornos anormais e influenciar o preço das ações, o estudo da relação entre dividendos e a volatilidade das ações é quase ausente em mercados emergentes (RASHID; RAHMAN, 2008). Conforme destacam Famá e lovine (2012), os estudos relacionados à política de dividendos no Brasil são recentes, com destaque a partir da década de 90 , período em que ocorreu a estabilização da economia brasileira. Ademais, observam-se tendências nos trabalhos sobre política de dividendos no Brasil, tais como: relevância da política de dividendos, confirmação da existência de problemas de agência, resultados conflitantes quanto à hipótese do efeito clientela; sinalização e tributação de dividendos (FAMÁ; IOVINE, 2012).

Nesse sentido, surge a seguinte situação problemática: qual o efeito da política de dividendos sobre a volatilidade do preço das ações no mercado acionário brasileiro? Diante do exposto, este artigo tem por objetivo verificar como a política de dividendos das organizações influencia a volatilidade do 
preço das ações negociadas na BM\&FBOVESPA. Essa análise da volatilidade é de fundamental importância, visto que, quanto mais elevada for à volatilidade de uma ação, maior será a oscilação do preço, resultando em um alto risco de se ganhar ou perder, ao negociar uma ação dessa magnitude.

Além desta, o presente artigo possui cinco partes. Na seguinte, apresenta-se a revisão de literatura, onde serão abordadas as teorias relacionadas à relevância ou não da política de dividendos e suas implicações, bem como as principais evidências empíricas entre a política de dividendos e a volatilidade das ações. Na terceira parte, abordar-se a metodologia. Na quarta, a análise dos resultados da pesquisa. Na quinta, a conclusão. E, por fim, as referências.

\section{REVISÃO DA LITERATURA}

Uma das maiores questões sobre a política de dividendos é sua influência ou não sobre as organizações e também se interfere nas atitudes dos investidores. Por causa dessa dificuldade em determinar objetivamente a influencia da política de dividendos, diversos estudos empíricos e teóricos foram desenvolvidos, para tentar explicar a relação entre a política de dividendos e fatores como o valor da empresa, o preço da ação ou o comportamento do investidor.

Recentemente, diferentes pesquisas, em âmbito internacional, têm como objeto de estudo a relação entre a política de dividendos e a variação no preço das ações, onde essa relação é observada pelo lado do risco (BASKIN, 1989; ALLEN; RACHIM, 1996; NISHAT; IRFAN, 2001; RASHID; RAHMAN, 2008; NAZIR et al., 2010; HUSSAINEY; MGBAME; CHIJOKE-MGBAME, 2011; HASHEMIJOO, ARDEKANI; YOUNESI, 2012), e não pelo lado do retorno, conforme comumente documentado na literatura. Teorias como a relevância/irrelevância da política de dividendos, da preferência por dividendos, da assimetria de informação, da sinalização, dentre outras, procuram explicar a relação entre dividendos e o preço das ações.

Miller e Modiglianni (1961) defendem que em um mercado perfeito, onde o comportamento do investidor é racional, a política de dividendos (atual e futura) é irrelevante para a avaliação de uma organização. Nesse sentido, a mesma seria avaliada apenas por suas oportunidades de investimento.

Nesse sentido, o pressuposto implícito do argumento da irrelevância dos dividendos consiste em defender que as empresas que pagam mais dividendos oferecem uma valorização menor, mas devem fornecer o mesmo retorno total para os acionistas, dada as suas características de risco e os fluxos de caixa de suas decisões de investimento. Portanto, se não há impostos, ou se os dividendos e ganhos de capital são tributados com a mesma taxa, os investidores devem ser indiferentes entre receber seus retornos como dividendos ou como valorização nos preços das ações (MILLER; MODIGLIANNI, 1961).

Por outro lado, defendendo-se a relevância, destacam-se os trabalhos de Lintner (1956) e Gordon (1959). Lintner (1956) argumenta que as decisões de dividendos representam o primeiro passo para muitas situações e demonstra que há uma crença, por parte dos administradores, na qual os acionistas preferem uma taxa razoavelmente estável e que o mercado estabelece um prêmio por essa estabilidade ou sobre um crescimento gradual na taxa de dividendos. Portanto, para atrair ou atender a demanda dos acionistas, as organizações procuram manter estável ou em um crescimento gradativo as suas políticas de dividendos.

Da mesma forma, o investidor, avesso ao risco e incerto quanto ao futuro, ao adquirir determinada ação estaria pagando por dividendos e/ou ganhos de capital, onde há a preferência pelo recebimento do dividendo à expectativa de ganho. Dessa maneira, empresas com grandes pagamentos de dividendos enfrentam menor risco, em termos da volatilidade do preço (GORDON, 1959). 
Para Black (1976), o pagamento de dividendos é uma maneira de transmitir informação que explicitamente não são realizadas pelos administradores. Nesse sentido, o aumento no pagamento de dividendos poderá sinalizar ao mercado um aumento no fluxo de caixa e, consequentemente, um bom desempenho. Por outro lado, uma diminuição poderá indicar ao mercado um mau desempenho da organização. Nesse sentido, é defendido que um aumento (diminuição) no pagamento de dividendos influencie positivamente (negativamente) o preço da ação.

Em contrapartida, o aumento no pagamento de proventos também poderá ser visto como negativo pelo mercado financeiro. Por exemplo:

(...) ao considerar uma empresa que nunca pagou dividendos no passado, mas que registrou um crescimento extraordinário e alto retorno sobre seus projetos. Quando esta empresa começar a pagar ou aumentar substancialmente os dividendos, seus acionistas podem considerar isto como um indicativo de que os projetos da empresa não são tão lucrativos como costumavam ser BRUNI et al. (2003, p. 5).

Em oura perspectiva, de acordo com a Teoria da Assimetria de Informação, na qual investidores não possuem as mesmas informações que os administradores das empresas, atitudes como a contração de dívida, distribuição (ou não) de dividendos ou emissão (recompra) de ações poderão ter significados diferentes para o investidor, o que afetará sua decisão de compra/venda e, consequentemente, o preço das ações (MYERS; MAJLUF, 1984).

Iquiapaza, Lamounier e Amaral (2008) analisaram o efeito da assimetria de informação sobre o pagamento de proventos de 178 empresas de capital aberto listadas na BM\&FBOVESPA. Utilizando um modelo de regressão censurado para análise dos dados, verificou-se que empresas com ADR (American Depositare Receipts) possuem menos assimetria de informação e, de acordo com a teoria da sinalização, uma relação inversa com o pagamento de proventos.

No mercado americano, os estudos que relacionam a política de dividendos e a volatilidade do preço das ações foram conduzidos por Harkavy (1953), Friend e Puckett, (1964), Litzenberger e Ramaswamy (1982), Fama e French (1988) e Baskin (1989), no entanto, esses estudos não são conclusivos.

Harkavy (1953) e Friend e Puckett (1964) constataram que existe uma tendência para os preços das ações estarem diretamente relacionados com a proporção dos lucros distribuídos e de associarem esse pagamento de maneira inversa com a incerteza do desempenho operacional futuro da organização. Ademais, Litzenberger e Ramaswamy (1982) verificaram a relação entre o dividend yield e o retorno das ações através de um modelo de precificação de ativos após impostos, onde encontraram uma relação positiva, porém não linear, entre as duas medidas.

Por outro lado, Baskin (1989) encontrou uma relação negativa e significante entre a política de dividendos e a volatilidade das ações. Em seu estudo, utiliza-se dos principais modelos que relacionam os dividendos aos preços das ações para analisar, em uma nova vertente, a influência do pagamento de dividendos sobre a volatilidade das ações. Adicionalmente, para verificar essa relação, Baskin (1989) sugere o uso de variáveis de controle para garantir a robustez da análise, em virtude destas variáveis influenciarem tanto o nível de pagamento de dividendos quanto a volatilidade do preço das ações, quais sejam: tamanho, volatilidade dos lucros, dívida de longo prazo e crescimento.

Fora do mercado americano, diferentes estudos procuraram analisar a relação entre a volatilidade das ações e o pagamento de dividendos com a utilização da metodologia utilizada por Baskin (1989) (ALLEN; RACHIM, 1996, NISHAT; IRFAN, 2001, RASHID; RAHMAN, 2008, NAZIR et al., 2010, HUSSAINEY; MGBAME; CHIJOKE-MGBAME, 2011, HASHEMIJOO; ARDEKANI; YOUNESI, 2012). 
No mercado australiano, Allen e Rachim (1996) verificaram que a política de dividendos não apresentou significância na explicação da volatilidade do preço das ações, encontrando, apenas, uma relação linear negativa e significante estatisticamente entre o índice payout e a volatilidade do preço das ações.

Nishat e Irfan (2001), no Paquistão, analisaram uma amostra de 160 empresas listadas na KSE (Karachi Stock Exchange), no período de 1981 a 2000. Utilizando a técnica de mínimos quadrados generalizados, verificaram que tanto o dividend yield quanto o índice payout apresentaram relação inversa com a volatilidade do preço das ações, conforme Baskin (1989). Ademais, as variáveis de controle apresentaram resultado semelhante ao de Allen e Rachim (1996).

Rashid e Rahman (2008), por meio da análise de regressão em cross-section, encontraram uma relação positiva, mas não significante, entre o dividend yield e a volatilidade. Nazir et al. (2010) analisaram 73 empresas, no período de 2003 a 2008, utilizando regressão em dados em painel, e encontraram uma relação positiva e significante entre o dividend yield e a volatilidade da empresas, no Paquistão.

Hussainey, Mgbame e Chijoke-Mgbame (2011) avaliaram a relação na bolsa de Londres, no período de 1998 a 2007, por meio de regressão múltipla e verificaram que tanto o dividend yield, quanto o índice de payout, apresentaram significância estatística. Entretanto, apenas o índice de payout apresentou-se inversamente relacionado à volatilidade das ações, contrariando os achados de Baskin (1989), fato este possivelmente causado por problemas de multicolinearidade. Por fim, Hashemijoo, Ardekani e Younesi (2012) encontraram, para o mercado de ações da Malásia, uma relação negativa e significante entre dividend yield e volatilidade do preço das ações.

\section{METODOLOGIA}

\subsection{AMOSTRA E TRATAMENTO DOS DADOS}

A amostra consistiu de todas as empresas não financeiras, pagadoras de dividendos e com ações listadas na Bolsa de Valores do Estado de São Paulo - BM\&FBOVESPA, no período de 2000 a 2012. Adicionalmente, para realizar a seleção das empresas, no caso de duplicidade de informação de uma mesma empresa, utilizou-se a classe da ação (preferencial ou ordinária) que apresentou maior liquidez em bolsa. Os dados necessários foram coletados do banco de dados da Economática.

Ademais, foram excluídas as empresas que não apresentaram todos os dados necessários para a pesquisa, bem como foram retirados os outliers, tendo em vista que esses dados apresentam comportamento discrepante com o restante da amostra. Para a identificação dos valores anormais, utilizou-se o procedimento de padronização em escore $Z$, bem como a análise gráfica.

De acordo com o Gráfico 1, existem 39 observações que se encontram acima de dois desvios da média. Contudo, antes de excluí-las, verificou-se se as referidas observações eram ponto de alavanca ou de influência e percebeu-se que apenas 13 observações não eram nem ponto de alavanca, nem de influência e, portanto, podiam ser excluídas. As outras 26 observações, apesar de ser um outlier, não foram excluída, por serem ponto de alavanca ou de influência. Dessa forma, na amostra final ficou composta por 914 observações. 


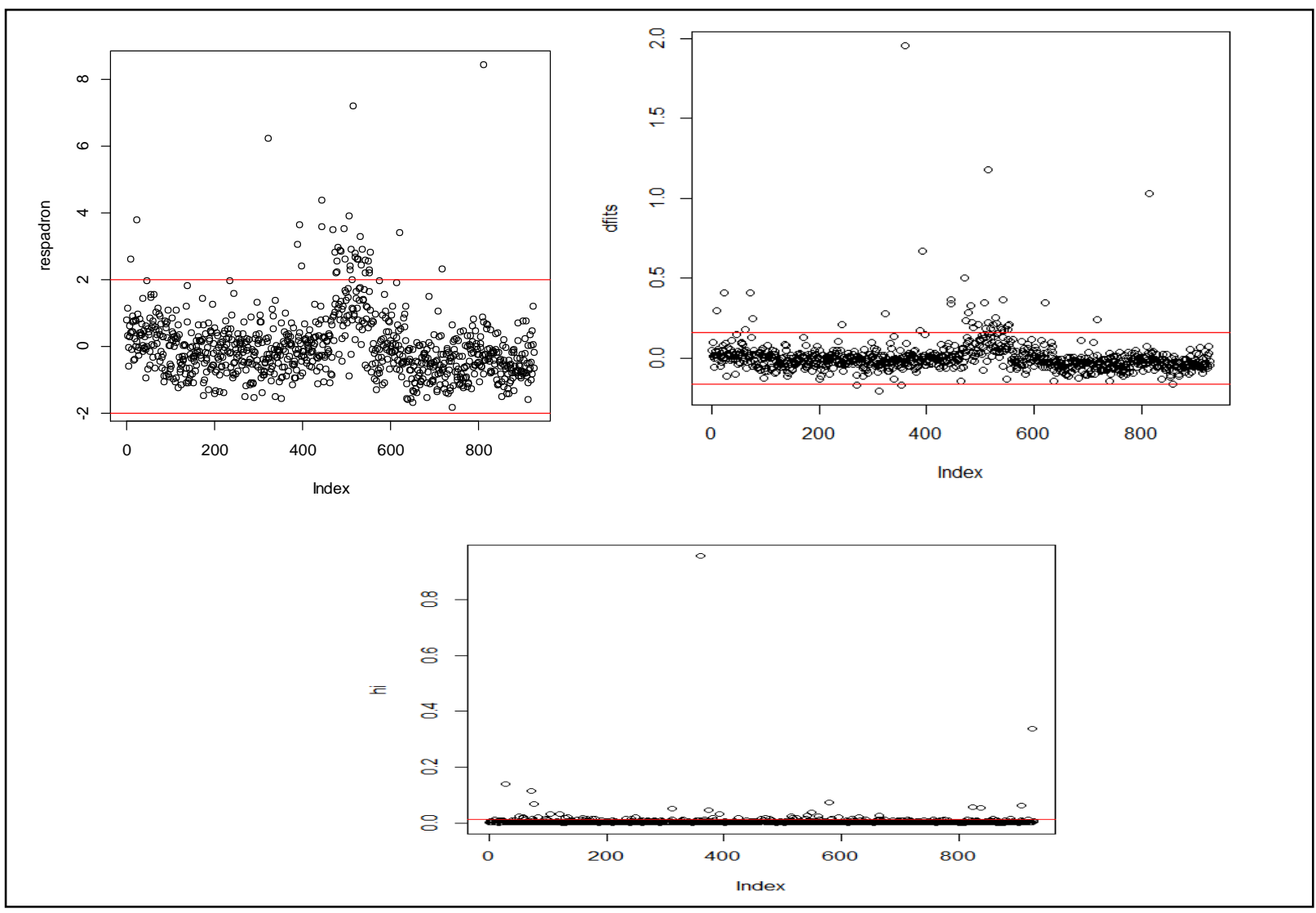

Gráfico 1 - Pontos Aberrantes, de Alavancagem e de Influência.

\subsection{MODELO ECONOMÉTRICO}

O modelo econométrico utilizado teve por base os trabalhos de Baskin (1989), Allen e Rachim (1996), Nishat e Irfan (2001), Rashid e Rahman (2008), Nazir et al. (2010), Hussainey, Mgbame e ChijokeMgbame (2011) e Hashemijoo, Ardekani e Younesi (2012). Para se mensurar a política de dividendos, utilizaram-se o dividend yield e o índice de payout. Para se avaliar o impacto da política de dividendos sobre a volatilidade das ações, o presente artigo utiliza técnicas de regressão por mínimos quadrados ordinários.

Para examinar a relação entre a política de dividendos e a volatilidade das ações, serão utilizadas duas variáveis explicativas: dividend yield e o índice payout. $\mathrm{O}$ dividend yield ou rendimento dos dividendos indica a remuneração do acionista realizada sobre o capital investido (valor pago pela empresa). Trata-se de um índice criado para medir a rentabilidade dos dividendos de uma empresa em relação ao preço de suas ações. Assim, quanto mais acentuado o dividend yield, maior será a política de distribuição de lucros. Por outro lado, o índice payout (índice de pagamento de dividendos) estabelece uma relação entre os dividendos pagos e os lucros de uma empresa. Adicionalmente, serão utilizadas como variáveis de controle a variável tamanho, a volatilidade dos lucros, a dívida de longo prazo e o crescimento dos ativos.

Tendo em vista que outros fatores, além do dividend yield e do índice payout, podem influenciar a volatilidade das ações, foram incluídas, na análise empírica, algumas variáveis de controle, de modo a garantir a robustez da análise. Tais variáveis são: tamanho, volatilidade dos lucros, dívida de longo 
prazo e crescimento. Assim, pretende-se avaliar a influência das variáveis dividend yield e o índice de payout sobre a volatilidade, por meio da Equação 1:

$\operatorname{Vol}_{i, \mathrm{t}}=\alpha_{0}+\alpha_{1}$ dyld $_{\mathrm{i}, \mathrm{t}}+\alpha_{2}$ pay $_{\mathrm{i}, \mathrm{t}}+\alpha_{3} \operatorname{tam}_{\mathrm{i}, \mathrm{t}}+\alpha_{4} \mathrm{evol}_{\mathrm{i}, \mathrm{t}}+\alpha_{5} \mathrm{dlp}_{\mathrm{i}, \mathrm{t}}+\alpha_{6} \mathrm{cresc}_{\mathrm{i}, \mathrm{t}}+\varepsilon_{\mathrm{i}}$

Onde:

Voli, $t$ = variável dependente, representada pela volatilidade dos preços da ação i no período $t$;

$D y / d i, t=$ variável independente, representada pelo dividend yield da empresa $\mathrm{i}$, no período $\mathrm{t}$;

Payi, $t$ = variável independente, representada pelo índice payout da empresa $\mathrm{i}$, no período $\mathrm{t}$;

Tami,$t=$ variável independente, representada pelo tamanho da empresa $\mathrm{i}$, no período $\mathrm{t}$;

Evoli, $t=$ variável independente, representada pela volatilidade do lucro da empresa i, no período t;

$D / p i, t=$ variável independente, representada pela dívida de longo prazo da empresa $\mathrm{i}$, no período $t$; Cresci, $t=$ variável independente, representada pelo crescimento do ativo da empresa i, no período t; $\varepsilon i, t$ = é o erro aleatório (ruído branco) da empresa i, no período t, com distribuição normal, média zero e variância constante.

Pela análise de estudos anteriores (BASKIN, 1989, ALLEN; RACHIM, 1996, NISHAT; IRFAN, 2001, RASHID; RAHMAN, 2008, NAZIR ET AL, 2010, HUSSAINEY; MGBAME; CHIJOKE-MGBAME, 2011), verificou-se que essas variáveis possuem impacto na explicação da oscilação do preço frente à distribuição de dividendos. Ademais, a variável tamanho poderá indicar que as grandes empresas apresentam maiores oportunidades de investimentos, resultando em baixo índice de dividendos pagos. Por outro lado, empresas maiores poderão também desenvolver uma política de altos dividendos, evitando que sua estratégia de atrair novos investidores seja imitada por empresas menores (IQUIAPAZA; LAMOUNIER; AMARAL, 2008).

No Brasil, em relação à dívida de longo prazo, verifica-se uma relação inversa entre endividamento e o pagamento de dividendos (IQUIAPAZA; BARBOSA; BRESSAN, 2005). Já a volatilidade dos lucros é uma proxy utilizada para mensurar a incerteza do investidos quanto ao desempenho futuro da organização. Por fim, verifica-se que o crescimento de ativos implica que empresas com alto crescimento são pagadoras de baixos dividendos (IQUIAPAZA; LAMOUNIER; AMARAL, 2008, FERREIRA JUNIOR; NAKAMURA; MARTIN; BASTOS, 2010).

Para a análise de regressão, basicamente, consideraram-se os valores do R2, os p-valores do teste $\mathrm{F}$ e do teste t. Segundo Brooks (2002), a análise do R2 mostra o poder de explicação da variável dependente pelas variáveis independentes. Já os p-valores dos testes $\mathrm{F}$ e $\mathrm{t}$ evidenciam se as variáveis independentes em conjunto e individualmente, respectivamente, são estatisticamente capazes de explicar o comportamento da variável dependente.

Em seguida, procederam-se aos testes dos pressupostos, no intuito de avaliar se o modelo analisado sofria alguma violação. O Quadro 1 evidencia a descrição da variáveis utilizadas na pesquisa.

Quadro 1 - Variáveis utilizadas

\begin{tabular}{|l|l|c|}
\hline \multicolumn{1}{|c|}{ Variável } & \multicolumn{1}{|c|}{ Descrição } & Efeito Esperado \\
\hline Volatilidade do preço & $\begin{array}{l}\text { É a variável dependente, mede as oscilações do preço das } \\
\text { ações frente às influências do mercado. A Volatilidade é } \\
\text { obtida pelo desvio padrão do logaritmo neperiano das } \\
\text { variações diárias da cotação de fechamento no período } \\
\text { escolhido }\end{array}$ & $\begin{array}{l}\text { Negativo } \\
\text { Dividend Yield }\end{array}$ \\
\hline É o dividendo pago por ação de uma empresa, dividido pelo \\
Índice Payout & $\begin{array}{l}\text { Consiste na quantidade do lucro distribuída por cada ação. } \\
\text { Calculado pela divisão dos dividendos por ação pelo lucro } \\
\text { por ação. }\end{array}$ & Negativo \\
\hline
\end{tabular}




\begin{tabular}{|l|l|c|}
\hline Tamanho & $\begin{array}{l}\text { É obtido pelo valor de mercado da empresa. Para seu } \\
\text { cálculo, foi utilizado o logaritmo natural. }\end{array}$ & Negativo \\
\hline Volatilidade dos Lucros & $\begin{array}{l}\text { É calculado razão entre o desvio padrão do lucro antes do } \\
\text { juros e imposto de renda (EBIT) dos cinco anos anteriores } \\
\text { ao período analisado e o ativo total. }\end{array}$ & Positivo \\
\hline Dívida de Longo Prazo & $\begin{array}{l}\text { Representa o exigível de longo prazo. Para inclusão nos } \\
\text { testes, foi calculada a razão entre a dívida de longo prazo e } \\
\text { o ativo total. }\end{array}$ & Positivo \\
\hline Crescimento do Ativo & $\begin{array}{l}\text { Medido pela variação do ativo total do inicio para o final do } \\
\text { ano. }\end{array}$ & Positivo \\
\hline
\end{tabular}

\section{ANÁLISE DOS RESULTADOS}

As estatísticas descritivas, apresentadas na Tabela 1, fornecem à média, o desvio padrão e os valores mínimos e máximos das variáveis.

Tabela 1 - Descrição das Variáveis

\begin{tabular}{ccccc}
\hline & Média & Desvio Padrão & Mínimo & Máximo \\
\hline \hline vol & 39,388 & 14,126 & 15,283 & 164,984 \\
dyld & 5,903 & 5,930 & 0,000 & 66,767 \\
pay & 0,781 & 0,9132 & $-36,551$ & 272,034 \\
tam & 15,096 & 1,550 & 9,160 & 19,879 \\
evol & 0,034 & 0,029 & 0,002 & 0,536 \\
dlp & 0,283 & 0,141 &, 000 & 0,790 \\
cresc & 0,180 & 0,327 & $-0,881$ & 3,840
\end{tabular}

Onde, vol = Volatilidade do preço; $d$ yld = Dividend Yield; pay = índice payout; tam = tamanho; evol = volatilidade dos lucros; dlp = dívida de longo prazo e cresc = crescimento dos ativos.

A Tabela 1 apresenta o detalhamento das variáveis que integram o modelo em questão. Diante disso, a variável vol (variável dependente), a qual representa as oscilações do preço das ações durante o ano, registrou um valor médio de aproximadamente 39,388 e desvio padrão de 14,126, variando entre o limite mínimo de 15,283 e máximo de 164,984. Com relação à descrição das variáveis independentes, verifica-se que rentabilidade média dos dividendos em relação ao preço da ação foi de 5,903\% com desvio padrão de 5,93, variando de 0 a 66,767. Já o índice payout, que mede a relação entre os dividendos distribuídos e o lucro líquido do exercício, oscila de um valor mínimo de 36,551 a um máximo de 272,034 , além disso, possui um valor médio de 0,781 , com desvio padrão de 0,9132 .

Outra variável incluída no modelo explicativo é o tamanho, representado pelo logaritmo natural do valor da empresa. Seu valor médio é 15,096 e desvio padrão de 1,55, com intervalo entre 9,16 e 19,879. A volatilidade dos lucros é a quarta variável utilizada para verificar a volatilidade do preço das ações, com média de 0,034 e desvio padrão de 0,029, com um intervalo entre 0,002 e máximo de 0,536 . Os valores da dívida de longo prazo variam de 0 até 0,79 , tendo valor médio igual a 0,283 e desvio padrão de 0,141 . Por fim, a variável crescimento dos ativos variou de $-0,881$ a 0,384 , com valor médio de 0,18 e desvio padrão de 0,327. 
Tabela 2 - Matriz de Correlação das Variáveis

\begin{tabular}{cccccccc}
\hline & vol & dyld & pay & tam & evol & dlp & cresc \\
\hline \hline Vol & 1 & & & & & & \\
Dyld & $-0,096^{*}$ & 1 & & & & & \\
Pay & $-, 0,036$ & $-0,002$ & 1 & & & & \\
Tam & $-0,236^{*}$ & $-0,052$ & $-0,012$ & 1 & & & \\
Evol & $-0,048$ & $0,117^{*}$ & $-0,009$ & $-0,083$ & 1 & & \\
Dlp & $0,101^{*}$ & $-0,048$ & 0,056 & $0,203^{*}$ & $-0,221^{*}$ & 1 & 1 \\
Cresc & $0,091^{*}$ & $-0,121^{*}$ & $-0,014$ & $0,065^{* *}$ & $-0,194^{*}$ & 0,056 & 1 \\
\hline
\end{tabular}

* Significativo a $1 \% ; * *$ Significativo a $5 \%$

A Tabela 2 apresenta a matriz de correlação entre as variáveis. Observa-se que a variável dependente possui relação significativa ao nível de $1 \%$ com a varável dividend yield, porém relação não significativa com a variável payout. Constata-se, também, que as variáveis dividend yield e índice payout são inversamente relacionadas com a volatilidade, como esperado, indicando que quanto maior o rendimento de dividendos, o índice de pagamento de dividendos menor será a oscilação do preço da ação e, consequentemente, menor o seu risco. No que diz respeito às variáveis de controle, observa-se que as variáveis tamanho, dívida de longo prazo e crescimento apresentaram relação significativa com a variável dependente, com o tamanho sendo negativamente relacionada e as variáveis dívida de longo prazo e crescimento positivamente relacionada, como esperado.

Ademais, pela análise de correlação entre as variáveis explicativas, observa-se que as variáveis explicativas apresentaram baixa correlação entre si, dando indícios de ausência de multicolinearidade. Observa-se uma relação negativa entre a política de dividendos e o crescimento de ativos, que corrobora com os achados de Iquiapaza, Lamounier e Amaral (2008) e Ferreira Junior et al. (2010), no entanto, apenas o dividend yield é significante a 1\%. Essa relação inversa sugere que a diminuição no rendimento dos dividendos é resultante da utilização de capital para investimentos no ativo.

Verifica-se, ainda, que a variável tamanho possui relação significante e positiva com a dívida de longo prazo, indicando que quanto maior a organização, mais elevada é a sua dívida. Por fim, a volatilidade do lucro apresenta correlação negativa e significante com a dívida de longo prazo e o crescimento dos ativos, o que representa uma maior oscilação no lucro das organizações com as mudanças no ativo.

A Tabela 3 apresenta os resultados da regressão referente à Equação 1, onde buscou-se analisar a relação entre a política de dividendos e a volatilidade da ação. De acordo com o painel B, observa-se que a regressão estimada apresentou significância estatística ao nível de $1 \%$, conforme $p$-value da estatística F. Obteve-se um coeficiente de determinação ajustado de 0,1052, evidenciando que $10,52 \%$ das variações na volatilidade das ações são explicadas pelas variáveis representativas da política de dividendos, representadas pelas variáveis dividend yield e índice payout, bem como pelas variáveis de controle, representadas pelo crescimento, volatilidade dos lucros, dívidas de longo prazo e tamanho. 
Tabela 3 - Regressão utilizando Dyld e Payout mais variáveis de controle

\begin{tabular}{ccccc}
\hline \hline \multicolumn{5}{c}{ Painel A } \\
\hline Variável Explicativa & Coeficiente & Erro padrão & Estatística t & p-valor \\
C & 73,791 & 6,914 & 10,672 & 0,000 \\
Dyld & $-0,219$ & 0,0896 & $-2,443$ & 0,015 \\
Pay & $-0,069$ & 0,0800 & $-0,869$ & 0,132 \\
Evol & $-8,680$ & 11,309 & $-0,768$ & 0,443 \\
Tam & $-2,508$ & 0,469 & $-5,346$ & 0,000 \\
Dlp & 14,136 & 4,033 & 3,505 & 0,000 \\
cresc & 3,458 & 1,163 & 2,974 & 0,003 \\
\hline \multicolumn{2}{c}{ Painel B } & Descrição & Valor \\
Descrição & Valor & Teste F (Estatística) & 17,77 \\
R2 & 0,1052 & Teste F (p-value) & 0,00 \\
\hline R2 ajustado & 0,0925 & \multicolumn{4}{c}{. }
\end{tabular}

*Erros padrão ajustados para heterocedasticidade, usando matriz robusta de Davidson e MacKinnon (HC3), uma vez que as hipóteses nulas de variâncias homocedasticas foi rejeitada, ao nível de $1 \%$, pelo teste de Breusch- Pagan.

**O Teste Jarque-Bera rejeitou a hipótese nula de distribuição normal, entretanto, de acordo com o teorema do limite central e considerando que foram utilizadas 914 observações, o pressuposto da normalidade pode ser relaxado (BROOKS, 2002). Adicionalmente, a correção de Newey-West aumenta o erro padrão, diminuindo a estatística t, tornando sua estimativa mais robusta.

***Para detectar a presença de multicolinearidade, fez-se uso do teste FIV (variance inflation factor). Obteve-se um FIV de 1,026, 1,004, 1,049, 1,010, 1,095 e 1,052, para as variáveis dyld, pay, tam, evol, dlp e cresc, concluindo-se pela inexistência de colinearidade (LEVINE; BERENSON; STEPHAN, 2000).

Adicionalmente, constata-se que as variáveis utilizadas para se mensurar a política de dividendos tiveram um efeito inverso sobre a volatilidade das ações, conforme esperado, indicando que o alto pagamento de dividendos reduz o risco e também influencia o preço da ação (GORDON, 1963). Entretanto, quanto à significância, o painel $\mathrm{A}$, da Tabela 1, por meio da estatística $\mathrm{t}$, indica que apenas o dividend yield apresentou-se significativo estatisticamente ao nível de $1 \%$.

No que diz respeito às variáveis de controle, observa-se que as variáveis crescimento, dívida de longo prazo e tamanho mostram-se significantes estatisticamente, as duas primeiras positivamente e a última negativamente, como esperado. Por outro lado, a variável volatilidade dos lucros não se mostrou significante estatisticamente e com sinal contrário ao esperado.

\section{CONCLUSÃO}

O objetivo deste estudo foi analisar o impacto da política de dividendos sobre a volatilidade do preço das ações. Para a realização do estudo, utilizou-se como amostra as empresas não financeiras, pagadoras de dividendos e listadas na BM\&FBOVESPA, no período de 2000 a 2012. A análise empírica é baseada em uma análise de regressão, por mínimos quadrados ordinários, entre a volatilidade da ação e a política de dividendos. Adicionalmente, em virtude da possibilidade de multicolinearidade, foram utilizadas as variáveis de controle tamanho, volatilidade do lucro, dívida de longo prazo e crescimentos dos ativos.

Utilizando o rendimento de dividendos e o índice payout para mensurar a política de dividendos, encontrou-se uma relação negativa e significante estatisticamente para o dividend yield, consistente 
com os achados de Hussainey, Mgbame e Chijoke-Mgbame (2011) e Hashemijoo, Ardekani e Younesi (2012). Conforme o esperado, verificou-se que o tamanho influenciou negativamente as oscilações do preço das ações, enquanto que a dívida de longo prazo e o crescimento dos ativos apresentaram um efeito direto sobre a volatilidade do preço das ações.

Por fim, partindo-se do pressuposto de relevância da política de dividendos para a organização, esta pesquisa contribui com novas evidências empíricas a respeito do assunto, tendo em vista que muitos dos estudos realizados têm sido direcionados à mudança da política de dividendos adotada pelas organizações, relacionando-a a lucros anormais ou ao retorno das ações. Ademais, um dos resultados mais importante deste estudo, que o rendimento de dividendos apresenta uma relação inversa com a volatilidade do preço das ações, fornece informações relevantes sobre o risco para os investidores que utilizam a estratégia de dividendos para investir no mercado acionário.

Devem-se levar em consideração algumas limitações da pesquisa, onde as conclusões do estudo são restritas à amostra, a técnica e o modelo econométrico utilizado. Sugere-se para pesquisas futuras a utilização de novas variáveis, bem como de outras técnicas estatísticas.

\section{REFERÊNCIAS}

ALLEN, D. E. e RACHIM, V. S. Dividend policy and stock price volatility: Australian Evidence. Journal of Applied Economics, Vol. 6, p. 175-188, 1996.

BASKIN, J. Dividend policy and the volatility of common stock. Journal of Portfólio Management, 15 (3), 19-25, 1989.

BLACK, F. The dividend puzzle. Journal of Portfolio Management, v.2, p. 5-8, 1976.

BROOKS, C. Introductory econometrics for finance. Cambridge: Cambridge University Press, 2002.

BRUNI, A. L., et.al. O Anúncio da distribuição de dividendos e Seu efeito sobre os preços das ações: um estudo empírico no brasil. In: Congresso USP de Controladoria e Contabilidade, 3, 2003, Anais... São Paulo, 2003.

FAMA, E. F.; FRENCH, K. R. Dividends Yields and Expected Stocks Returns. Journal of Financial Economics, v. 22, n.1, p. 3-25, 1988.

FAMÁ, R.; IOVINE, A. O que revelam os estudos publicados no Brasil sobre política de dividendos? RAE (Impresso), v. 52, p. 1-15, 2012.

FERREIRA JUNIOR, W. O; NAKAMURA, W. T; MARTIN, D. M. L, BASTOS, D. D. Evidências empíricas dos fatores determinantes das políticas de dividendos das firmas listadas na Bovespa. Revista FACEF Pesquisa - Desenvolvimento e Gestão, v. 13, n. 2, p. 190 - 203, 2010.

FRIEND, I.; PUCKETT, M. Dividends and stock prices. American Economic Review, v.54, n.5, p. 656682, 1964.

GORDON, M. J. Dividends, earnings and stock prices. Review of Economics and Statistics, v.41, p. 99105, 1959.

GORDON, M. J. Optimal investment and financing policy. The Journal of finance. v.18, n.2, p.264-272, 1963.

HARKAVY, $O$. The relation between retained earnings and common stock prices for large, listed corporations. Journal of Finance, v.8, n.3, p. 283-297, 1953.

HASHEMIJOO, M; ARDEKANI, A. F; YOUNESI, N. The Impact of Dividend Policy on Share Price Volatility in the Malaysian Stock Market. Journal of Business Studies Quarterly, vol. 4, No. 1, p. 111-129, 2012. 
HUSSAINEY, K; MGBAME, C. O.; CHIJOKE-MGBAME, A. M. Dividend policy and share price volatility: UK evidence. Journal of Risk Finance, v.12, n.1, p. 57-68, 2011.

IQUIAPAZA, R. A; BARBOSA, F. V; BRESSAN, A. A. Dividendos: plano real, imposto de renda e sinalização nas empresas listadas na Bovespa: 1986 a 2003.In: Encontro Brasileiro de Finanças, 5, 2005, São Paulo. Anais eletrônicos... São Paulo: SBFIN, 2005.

IQUIAPAZA, R. A.; LAMOUNIER, W. M.; AMARAL, H. F. Asymmetric information and dividends payout at the São Paulo Stock Exchange. Advances in Scientific and Applied Accounting, v. 1, p. 1-15, 2008.

LEAL, R. P. C.; SAITO, R. Finanças Corporativas no Brasil. RAE-eletrônica, v. 2, n. 2, jul-dez/2003.

LEVINE, D. M.; BERENSON, M. L.; STEPHAN, D. Estatística: teoria e aplicações. Rio de Janeiro: LTC, 2000.

LINTER, J. Distributions of incomes of corporations among dividends, retained earnings and taxes. American economic Review, v.46, n.1, p. 97-113, 1956.

LITZENBERGER, R. H.; RAMASWAMY, K. The effects of dividends on common stock prices tax effects or information effects? Journal of Finance, v.37, p. 429-443, 1982.

MILLER, M.; MODIGLIANI, F. Dividend policy, growth, and valuation of shares. Journal of Business, v.34, p. 411-433, 1961.

MOTA, D. C.; EID JUNIOR, W. Distribuindo os Lucros. GV-executivo, v. 6, n. 3, maio-jun, 2007.

MYERS, S, C.; MAJLUF, N. S. Corporate financing and investiment decisions when firms have information that investors do not have. Journal of Financial Economics, v.13, p. 187-221, 1984.

NAZIR, M. S, et. al. Determinants of stock price volatility in Karachi Stock Exchange: The Mediating Role of Corporate Dividend Policy. International Research Journal of Finance and Economics, v.55, p. 100-107, 2010.

NISHAT, M.; IRFAN, C. M. Dividend policy and stock price volatility in Pakistan. In: Annual General Meeting And Conference Of The Pakistan Society Of Development Economists, 19., 2004, Paquistão. 2001.

RASHID, A.; RAHMAN, A. Z. .Dividend policy and stock price volatility: Evidence from Bangladesh. Journal of Applied Business and Economics, v.8, n.4, p. 71-81, 2008. 